This item was submitted to Loughborough's Research Repository by the author.

Items in Figshare are protected by copyright, with all rights reserved, unless otherwise indicated.

\title{
Post-local buckling-driven delamination in bilayer composite beams
}

PLEASE CITE THE PUBLISHED VERSION

\section{PUBLISHER}

(C) the authors

\section{VERSION}

VoR (Version of Record)

\section{PUBLISHER STATEMENT}

This work is made available according to the conditions of the Creative Commons Attribution-NonCommercialNoDerivatives 4.0 International (CC BY-NC-ND 4.0) licence. Full details of this licence are available at: https://creativecommons.org/licenses/by-nc-nd/4.0/

\section{LICENCE}

CC BY-NC-ND 4.0

\section{REPOSITORY RECORD}

Wang, Simon, Christopher M. Harvey, Bin Wang, and Andrew Watson. 2017. "Post-local Buckling-driven Delamination in Bilayer Composite Beams”. figshare. https://hdl.handle.net/2134/26716. 


\section{Post-Local Buckling Driven Delamination in Bilayer Composite Beams}

Simon Wang, Christopher Harvey, Bin Wang, Andrew Watson

s.wang@lboro.ac.uk, c.m.harvey@lboro.ac.uk

Department of Aeronautical \& Automotive Engineering

Loughborough University, LE11 3TU UK 


\section{Contents}

- Motivation and Aim

- Problem definition

- Delamination energy release rates (EER)

- Motivation and Aim

- Analytical Development

- Experimental validation

- Conclusions 


\section{Motivation and Aim}

- Problem definition

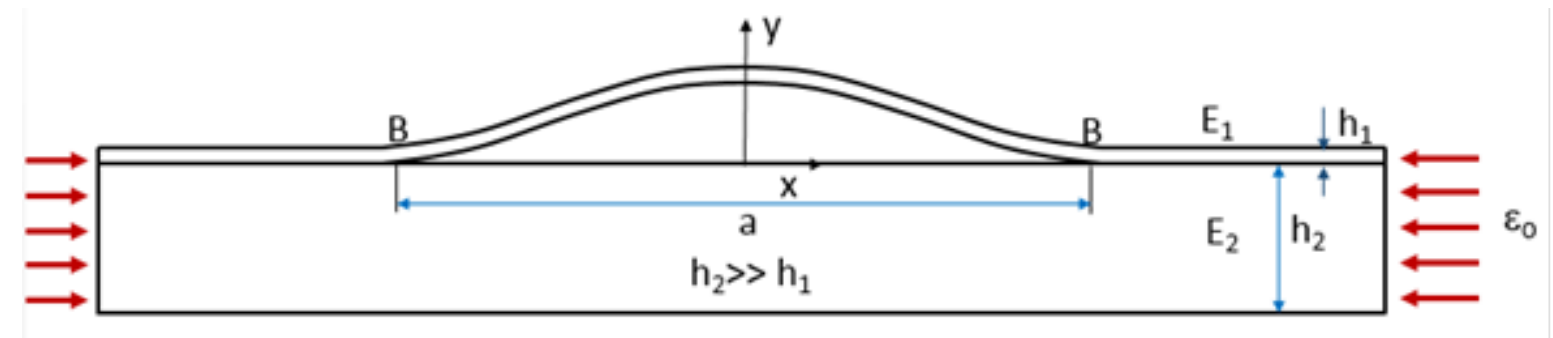

Fig. 1. A post-local buckled bilayer composite beam due to delamination under compression. 


\section{Motivation and Aim}

\section{- Problem definition}

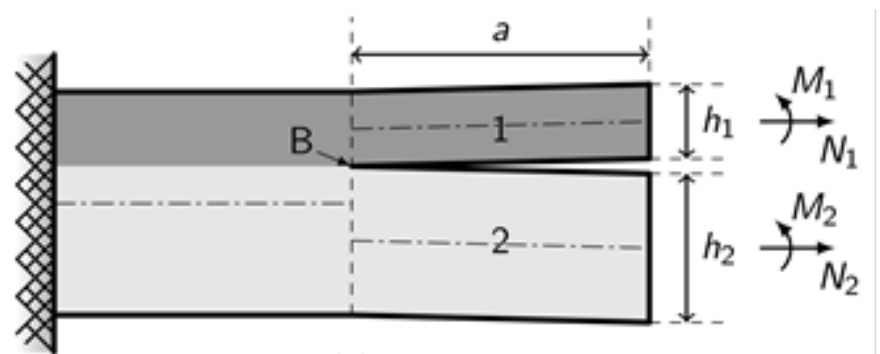

(a)

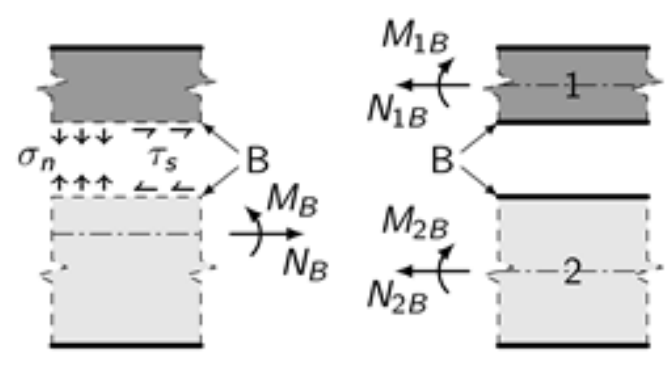

(b)

Fig. 2. Delamination tip internal forces and moments. 


\section{Motivation and Aim}

- Delamination energy release rates (ERRs)

$$
\begin{aligned}
G & =\frac{1}{2 b^{2}}\left(\frac{M_{1 B}{ }^{2}}{D_{1}^{*}}+\frac{M_{2 B}{ }^{2}}{D_{2}^{*}}-\frac{M_{B}{ }^{2}}{D^{*}}+\frac{N_{1 B}{ }^{2}}{A_{1}^{*}}+\frac{N_{2 B}{ }^{2}}{A_{2}^{*}}-\frac{N_{B}{ }^{2}}{A^{*}}-\frac{2 B_{1} M_{1 B} N_{1 B}}{B_{1}^{*}}-\frac{2 B_{2} M_{2 B} N_{2 B}}{B_{2}^{*}}+\frac{2 B M_{B} N_{B}}{B^{*}}\right) \\
& =\left\{M_{1 B}, M_{2 B}, N_{1 B}, N_{2 B}\right\}[C]\left\{M_{1 B}, M_{2 B}, N_{1 B}, N_{2 B}\right\}
\end{aligned}
$$

- ERR partitions GI and GII 


\section{Motivation and Aim}

\section{- Euler beam partitions:}

$$
\begin{aligned}
& G_{I E}=c_{I E}\left(M_{1 B}-\frac{M_{2 B}}{\beta_{1}}-\frac{N_{1}}{\beta_{2}}-\frac{N_{2}}{\beta_{3}}\right)\left(M_{1 B}-\frac{M_{2 B}}{\beta_{1^{\prime}}}-\frac{N_{1}}{\beta_{2^{\prime}}}-\frac{N_{2}}{\beta_{3^{\prime}}}\right) \\
& G_{I I E}=c_{I I E}\left(M_{1 B}-\frac{M_{2 B}}{\theta_{1}}-\frac{N_{1}}{\theta_{2}}-\frac{N_{2}}{\theta_{3}}\right)\left(M_{1 B}-\frac{M_{2 B}}{\theta_{1^{\prime}}}-\frac{N_{1}}{\theta_{2^{\prime}}}-\frac{N_{2}}{\theta_{3^{\prime}}}\right)
\end{aligned}
$$

- Timoshenko beam partitions:

$$
G_{I T}=c_{I T}\left(M_{1 B}-\frac{M_{2 B}}{\beta_{1}}-\frac{N_{1}}{\beta_{2}}-\frac{N_{2}}{\beta_{3}}\right)^{2} \quad G_{I I T}=c_{I I T}\left(M_{1 B}-\frac{M_{2 B}}{\theta_{1}}-\frac{N_{1}}{\theta_{2}}-\frac{N_{2}}{\theta_{3}}\right)^{2}
$$

- 2D elasticity partitions:

$$
G_{I 2 D}=C_{I 2 D}\left(M_{1 B}-\frac{M_{2 B}}{\beta_{12 D}}-\frac{N_{1}}{\beta_{22 D}}-\frac{N_{2}}{\beta_{32 D}}\right)^{2} \quad G_{I I 2 D}=C_{I I 2 D}\left(M_{1 B}-\frac{M_{2 B}}{\theta_{12 D}}-\frac{N_{1}}{\theta_{22 D}}-\frac{N_{2}}{\theta_{32 D}}\right)^{2}
$$




\section{Motivation and Aim}

- Motivation and Aim:

Extensive experimental tests $[1,2]$ have validated that Euler beam partitions give very accurate predictions of fracture toughness. The present work aims to validate if it still gives accurate predictions for post-local buckling driven delamination.

[1] Harvey CM, Wang S. Experimental assessments of mixed-mode partition theories. Composite Structures 94 (2012), 2057-67.

[2] Harvey CM, Eplett MR, Wang S. Experimental assessments of mixed-mode partition theories for generally laminated composite beams. Composite Structures 124 (2015), 10-18. 


\section{Analytical Development}

- Post-local buckling mode shape

$$
V_{1}\left(x_{1}\right)=A \frac{1}{2}\left(\cos \left(\frac{2 \alpha \pi x_{1}}{a}\right)-\cos (\alpha \pi)\right)
$$

- Critical local buckling strain

$$
\varepsilon_{c}=\frac{(\alpha \pi)^{2}}{3}\left(\frac{h_{1}}{a}\right)^{2}
$$

- Amplitude $A$

$$
\begin{gathered}
\left(1-\varepsilon_{c}\right) a / 2=\int d s=\int_{0}^{\left(1-\varepsilon_{0}\right) a / 2}\left[1+\left(d V_{1} / d x_{1}\right)^{2}\right]^{1 / 2} d x_{1} \approx \int_{0}^{a / 2}\left[1+\left(d V_{1} / d x_{1}\right)^{2}\right]^{1 / 2} d x_{1} \\
A=1.052 \varepsilon_{a}^{1 / 2} 2 a /(\alpha \pi)
\end{gathered}
$$




\section{Analytical Development}

\section{Post-local buckling total ERR G}

$$
G=\frac{1}{2 E_{1} b}\left[\frac{M_{1 B}^{2}}{I_{1}}+\frac{N_{1 B}^{2}}{A_{1}}+\frac{N_{2 B}^{2}}{A_{2 e}}-\frac{N_{3 B}^{2}}{A_{3 e}}\right] \quad G=\frac{1}{2} E_{1} h_{1} \varepsilon_{a}\left(4 c_{\alpha}^{2} \varepsilon_{c}+\lambda \varepsilon_{a}\right)
$$

Euler beam partitions

$$
G_{I E}=E_{1} h_{1} c_{\alpha} \varepsilon_{c}^{1 / 2} \varepsilon_{a}\left(2 c_{\alpha} \varepsilon_{c}^{1 / 2}-\sqrt{3} \lambda \varepsilon_{a}^{1 / 2}\right) \quad G_{I I E}=E_{1} h_{1} \lambda \varepsilon_{a}^{3 / 2}\left(\sqrt{3} c_{\alpha} \varepsilon_{c}^{1 / 2}+1 / 2 \varepsilon_{a}^{1 / 2}\right)
$$

\section{Timoshenko beam partitions}

$G_{I T}=\frac{1}{2(1+3 \lambda)} E_{1} h_{1} \varepsilon_{a}\left(2 c_{\alpha} \varepsilon_{c}^{1 / 2}-\sqrt{3} \lambda \varepsilon_{a}^{1 / 2}\right)^{2} \quad G_{I I T}=\frac{\lambda}{2(1+3 \lambda)} E_{1} h_{1} \varepsilon_{a}\left(2 \sqrt{3} c_{\alpha} \varepsilon_{c}^{1 / 2}+\varepsilon_{a}^{1 / 2}\right)^{2}$

2D elasticity partitions

$$
\begin{gathered}
G_{I 2 D}=\frac{12+2.697^{2} \lambda}{6(4.450+2.697 \lambda)^{2}} E_{1} h_{1} \varepsilon_{a}\left(4.450 c_{\alpha} \varepsilon_{c}^{1 / 2}-\sqrt{3} \lambda \varepsilon_{a}^{1 / 2}\right)^{2} \\
G_{I I 2 D}=\frac{\left(12 \lambda+4.450^{2}\right) \lambda}{6(4.450+2.697 \lambda)^{2}} E_{1} h_{1} \varepsilon_{a}\left(2.697 c_{\alpha} \varepsilon_{c}^{1 / 2}+\sqrt{3} \varepsilon_{a}^{1 / 2}\right)^{2}
\end{gathered}
$$




\section{Experimental Validation}

- The experimental data from the work

[8] Kutlu Z, Zhang KK. Composite panels containing multiple through-the -width delaminations and subjected to compression Part 1: Analysis. Composite Structures 31 (1995), 273-96.

[9] Kutlu Z, Zhang KK. Composite panels containing multiple through-the -width delaminations and subjected to compression Part 2: Experiments and verification. Composite Structures 31 (1995), 297-314. 


\section{Experimental Validation}

- Table 1. Configurations of two composite panels containing a single through-the-width delamination.

\begin{tabular}{|c|c|c|c|c|}
\hline Cases & Lay-ups & $\begin{array}{c}a \\
(\mathrm{~mm})\end{array}$ & $\begin{array}{c}h_{1} \\
(\mathrm{~mm})\end{array}$ & $\begin{array}{c}\text { Thickness } h \\
(\mathrm{~mm})\end{array}$ \\
\hline 1 & {$\left[0_{4} / 0_{12} / 0_{4}\right]$} & 38.1 & 0.518 & 2.59 \\
\hline 2 & {$\left[0_{4} / 0_{12} / 0_{4}\right]$} & 19.05 & 0.508 & 2.54 \\
\hline
\end{tabular}

Correction factor: $\alpha^{2}=11.738 *\left(\frac{h_{1}}{a}\right)^{2}-3.6654 *\left(\frac{h_{1}}{a}\right)+0.9755$

Failure criterion: $\quad f\left(G_{I}, G_{I I}, G_{I C}, G_{I C}\right)=\frac{G_{I}}{G_{I C}}+\frac{G_{I I}}{G_{I I C}}-1=0$

$$
E_{1}=139.3 \mathrm{MPa}, G_{I c}=0.0876 \mathrm{~N} / \mathrm{mm}, G_{\text {IIc }}=0.3152 \mathrm{~N} / \mathrm{mm}
$$




\section{Experimental Validation}

- Table 2. Verification of total ERR G for case 1

\begin{tabular}{|c|c|c|c|}
\hline \multirow{2}{*}{$10^{3} \varepsilon_{0}$} & \multicolumn{3}{|c|}{$G[\mathrm{~N} / \mathrm{mm}]$} \\
\cline { 2 - 4 } & Work [5] & Eq. (23) & Work [2,3] \\
\hline 1.00 & 0.0426 & 0.0445 & 0.0402 \\
\hline 1.20 & 0.0662 & 0.0682 & 0.0646 \\
\hline 1.40 & 0.0924 & 0.0945 & 0.0921 \\
\hline 1.60 & 0.1208 & 0.1231 & 0.1225 \\
\hline 1.80 & 0.1499 & 0.1540 & 0.1559 \\
\hline 2.00 & 0.1830 & 0.1872 & 0.1921 \\
\hline 2.20 & 0.2106 & 0.2227 & 0.2311 \\
\hline 2.40 & 0.2454 & 0.2605 & 0.2731 \\
\hline
\end{tabular}




\section{Experimental Validation}

- Table 3. Verification of total ERR G for case 2

\begin{tabular}{|c|c|c|c|}
\hline \multirow{2}{*}{$10^{3} \varepsilon_{0}$} & \multicolumn{3}{|c|}{$G[\mathrm{~N} / \mathrm{mm}]$} \\
\cline { 2 - 4 } & Work [5] & Eq. (23) & Work [2,3] \\
\hline 2.20 & 0.0345 & 0.0410 & 0 \\
\hline 2.30 & 0.0667 & 0.0737 & 0.0002 \\
\hline 2.40 & 0.0965 & 0.1056 & 0.0202 \\
\hline 2.50 & 0.1355 & 0.1398 & 0.0545 \\
\hline 2.60 & 0.1726 & 0.1732 & 0.0887 \\
\hline 2.70 & 0.2130 & 0.2069 & 0.1231 \\
\hline
\end{tabular}




\section{Experimental Validation}

- Table 4 Analytical prediction of propagation behaviour for case 1 panel

\begin{tabular}{|c|c|c|c|c|c|c|c|c|c|}
\hline \multirow{2}{*}{$10^{3} \varepsilon_{0}$} & \multicolumn{3}{|c|}{ Euler } & \multicolumn{3}{c|}{ Timoshenko } & \multicolumn{3}{|c|}{ 2D Elasticity } \\
\cline { 2 - 10 } & $\mathrm{a}$ & $f$ & $\begin{array}{c}G_{I I} / G \\
(\%)\end{array}$ & $\begin{array}{c}\mathrm{a} \\
{[\mathrm{mm}]}\end{array}$ & $f$ & $G_{I I} / G(\%)$ & $\begin{array}{c}\mathrm{a} \\
{[\mathrm{mm}]}\end{array}$ & $f$ & $\begin{array}{c}G_{I I} / G \\
(\%)\end{array}$ \\
\hline 0.6 & 38.1 & $<0$ & 17.6 & 38.1 & $<0$ & 79.8 & 38.1 & $<0$ & 43.0 \\
\hline 1.6 & 38.1 & $<0$ & 92.4 & 38.1 & $<0$ & 99.8 & 38.1 & $<0$ & 82.1 \\
\hline 2.0 & 38.1 & $<0$ & 100 & 38.1 & $<0$ & 100 & 38.1 & $<0$ & 87.4 \\
\hline $\mathbf{2 . 3 7}$ & 38.1 & $<0$ & 100 & 38.1 & $<0$ & 100 & 38.1 & 0 & 90.7 \\
\hline 2.42 & 38.1 & $<0$ & 100 & 38.1 & $<0$ & 100 & 39.1 & 0 & 92.0 \\
\hline $\mathbf{2 . 6 7}$ & 38.1 & 0 & 100 & 38.1 & 0 & 100 & 45.1 & 0 & 97.1 \\
\hline 2.69 & 39.1 & 0 & 100 & 39.1 & 0 & 100 & 45.6 & 0 & 97.3 \\
\hline 2.76 & 43.6 & 0 & 100 & 43.6 & 0 & 100 & 48.1 & 0 & 98.4 \\
\hline $\mathbf{2 . 8 2}$ & 48.1 & 0 & 100 & 48.1 & 0 & 100 & 50.8 & 0 & 99.2 \\
\hline $\mathbf{2 . 8 6}$ & 50.8 & 0 & 100 & 50.8 & 0 & 100 & - & - & - \\
\hline
\end{tabular}




\section{Experimental Validation}

- Fig. 3. Analytical prediction of propagation behaviour for case 1 panel

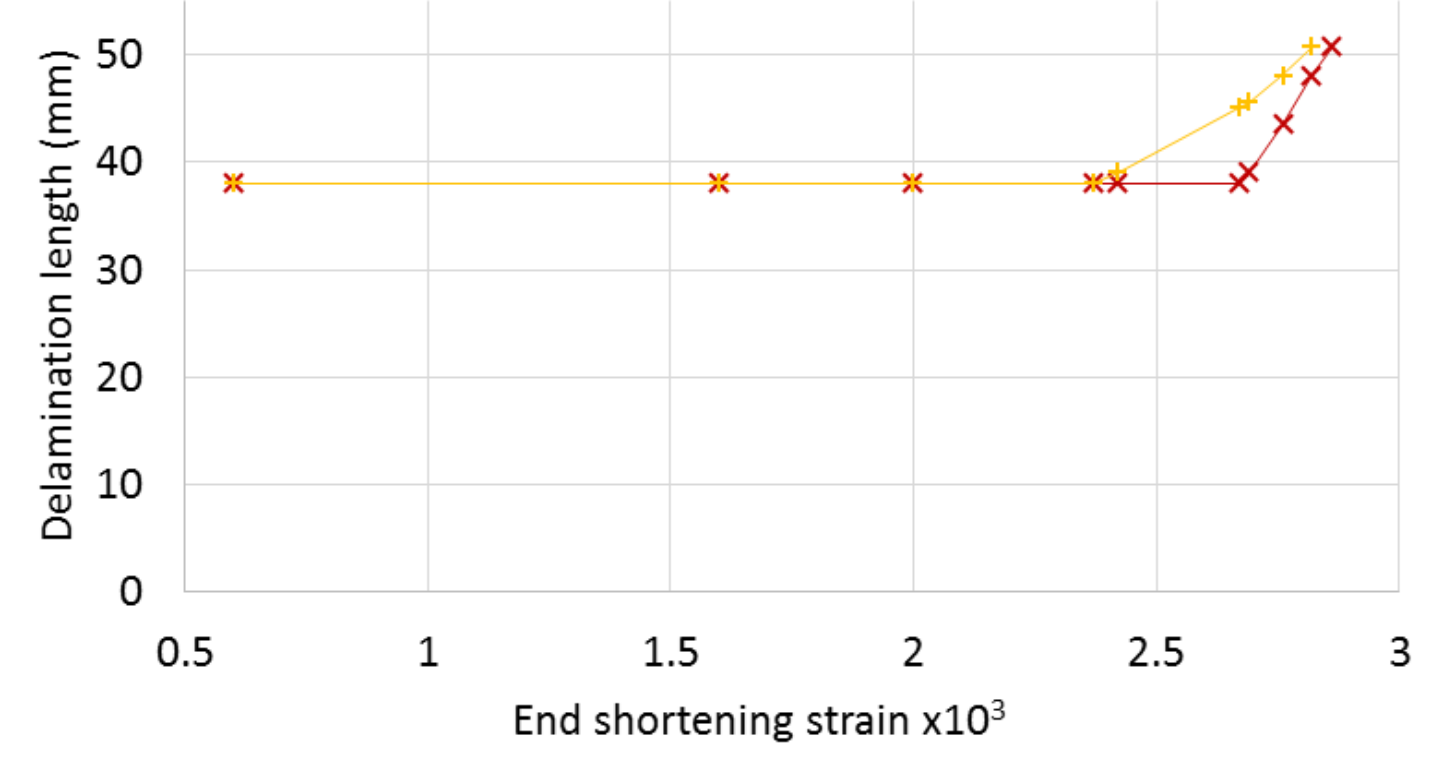

- Euler \& Timoshenko + 2D Elasticity 


\section{Experimental Validation}

- Fig. 4. Experimental validation of the analytical prediction for case 1 panel

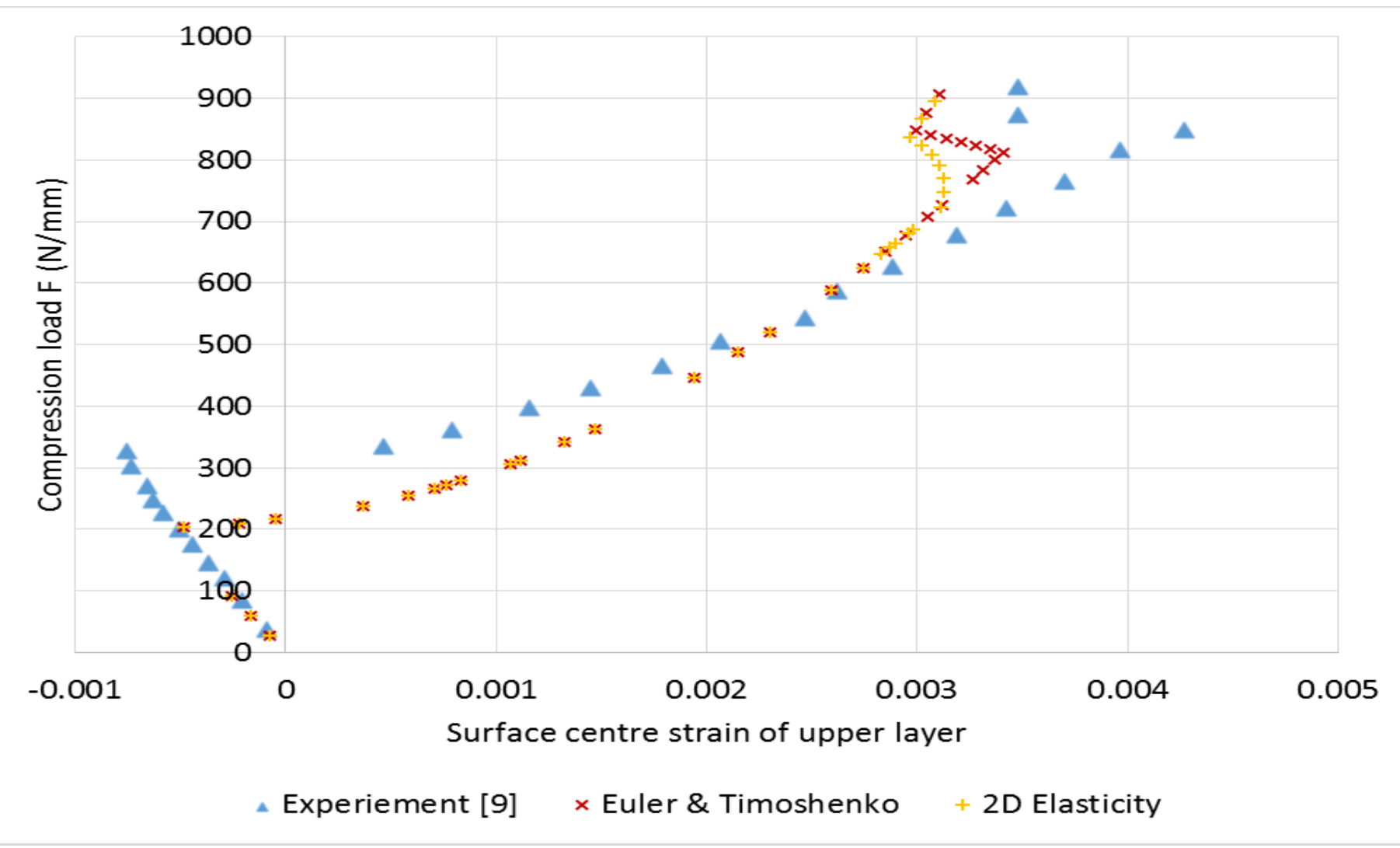




\section{Experimental Validation}

\section{- Fig. 5. Experimental validation of the analytical prediction for case 1 panel}

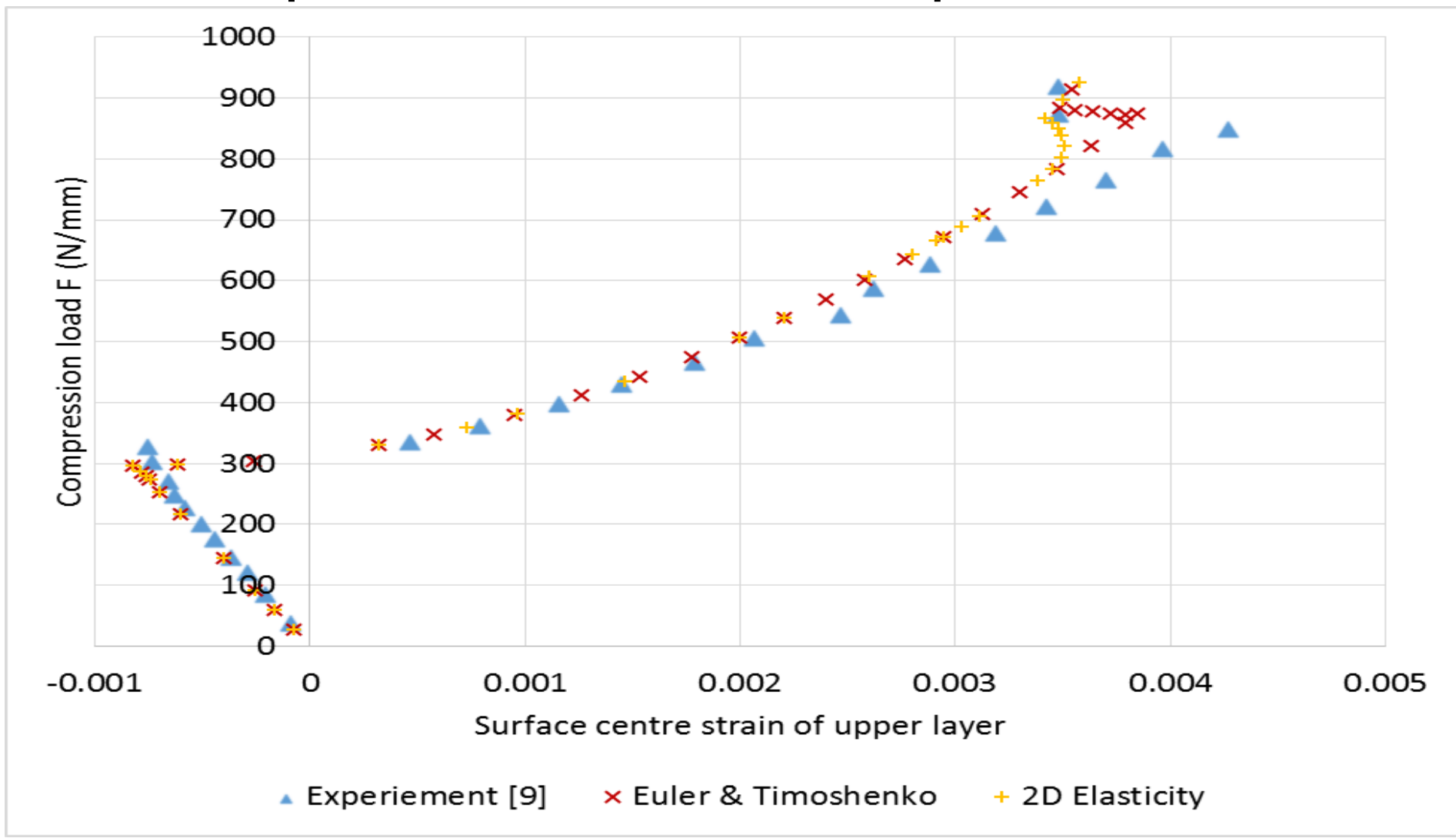




\section{Experimental Validation}

\section{- Table 5 Analytical prediction of propagation behaviour for case 2 panel}

\begin{tabular}{|c|c|c|c|c|c|c|c|c|c|}
\hline \multirow[b]{2}{*}{$10^{3} \varepsilon_{0}$} & \multicolumn{3}{|c|}{ Euler } & \multicolumn{3}{|c|}{ Timoshenko } & \multicolumn{3}{|c|}{ 2D Elasticity } \\
\hline & $\begin{array}{c}\mathrm{a} \\
{[\mathrm{mm}]}\end{array}$ & $f$ & $\begin{array}{c}G_{I I} / G \\
(\%)\end{array}$ & $\begin{array}{c}\mathrm{a} \\
{[\mathrm{mm}]}\end{array}$ & $f$ & $\begin{array}{c}G_{I I} / G \\
(\%)\end{array}$ & $\begin{array}{c}\mathrm{a} \\
{[\mathrm{mm}]}\end{array}$ & $f$ & $\begin{array}{c}G_{I I} / G \\
(\%)\end{array}$ \\
\hline 2.12 & 19.05 & $<0$ & 9.80 & 19.05 & $<0$ & 76.0 & 19.05 & $<0$ & 38.5 \\
\hline 2.40 & 19.05 & $<0$ & 28.7 & 19.05 & $<0$ & 84.6 & 19.05 & $<0$ & 49.3 \\
\hline 2.42 & 19.05 & $\geq 0$ & 29.5 & 19.05 & $<0$ & 85.0 & 19.05 & $<0$ & 49.8 \\
\hline 2.43 & 30.70 & 0 & 94.6 & 19.05 & $<0$ & 85.1 & 19.05 & $<0$ & 50.0 \\
\hline 2.48 & 31.00 & 0 & 96.5 & 19.05 & $<0$ & 86.0 & 19.05 & $<0$ & 51.2 \\
\hline 2.50 & 31.10 & 0 & 97.3 & 19.05 & $<0$ & 86.3 & 19.05 & $\geq 0$ & 51.7 \\
\hline 2.52 & 31.12 & 0 & 97.8 & 19.05 & $<0$ & 86.6 & 40.05 & 0 & 94.0 \\
\hline 2.60 & 31.30 & 0 & 99.9 & 19.05 & $<0$ & 87.7 & 41.65 & 0 & 95.4 \\
\hline 2.62 & 32.50 & 0 & 100 & 19.05 & $<0$ & 87.9 & 42.00 & 0 & 95.7 \\
\hline 2.68 & 36.05 & 0 & 100 & 19.05 & $<0$ & 88.7 & 43.60 & 0 & 96.8 \\
\hline 2.74 & 40.05 & 0 & 100 & 19.05 & $<0$ & 89.9 & 45.20 & 0 & 97.7 \\
\hline 2.80 & 43.30 & 0 & 100 & 19.05 & $<0$ & 90.0 & 47.05 & 0 & 98.5 \\
\hline 2.82 & 45.00 & 0 & 100 & 19.05 & $\geq 0$ & 90.1 & 48.10 & 0 & 98.8 \\
\hline 2.83 & 45.50 & 0 & 100 & 45.05 & 0 & 100 & 48.50 & 0 & 98.9 \\
\hline 2.84 & 46.00 & 0 & 100 & 46.05 & 0 & 100 & 48.90 & 0 & 99.0 \\
\hline 2.86 & 47.50 & 0 & 100 & 47.05 & 0 & 100 & 49.70 & 0 & 99.2 \\
\hline 2.88 & 48.50 & 0 & 100 & 49.05 & 0 & 100 & 50.08 & 0 & 99.4 \\
\hline 2.90 & 50.08 & 0 & 100 & 50.08 & 0 & 100 & - & - & - \\
\hline
\end{tabular}




\section{Experimental Validation}

- Fig. 6. Analytical prediction of propagation behaviour for case 2 panel

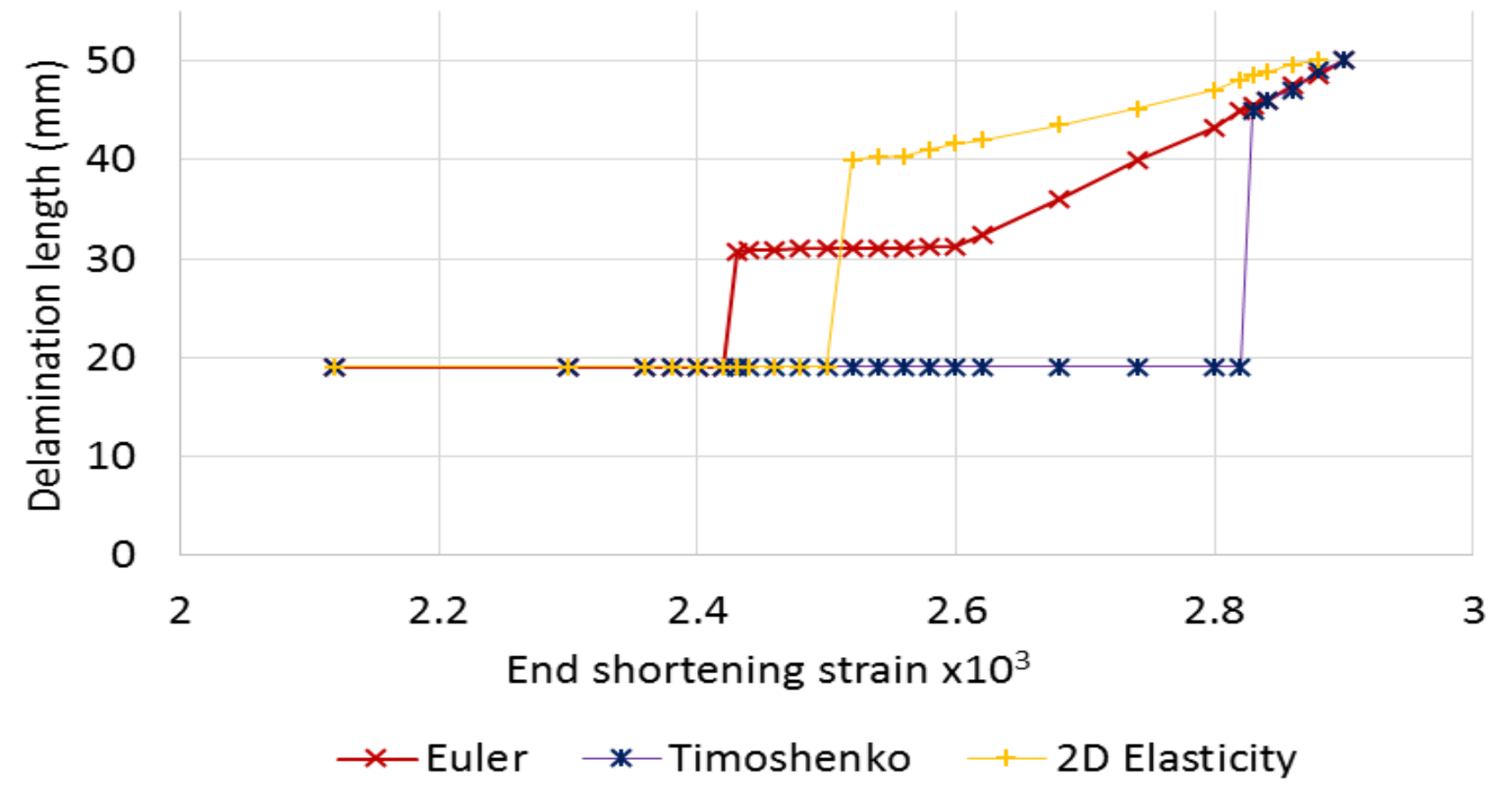




\section{Experimental Validation}

- Fig. 7. Experimental validation of the analytical prediction for case 2 panel

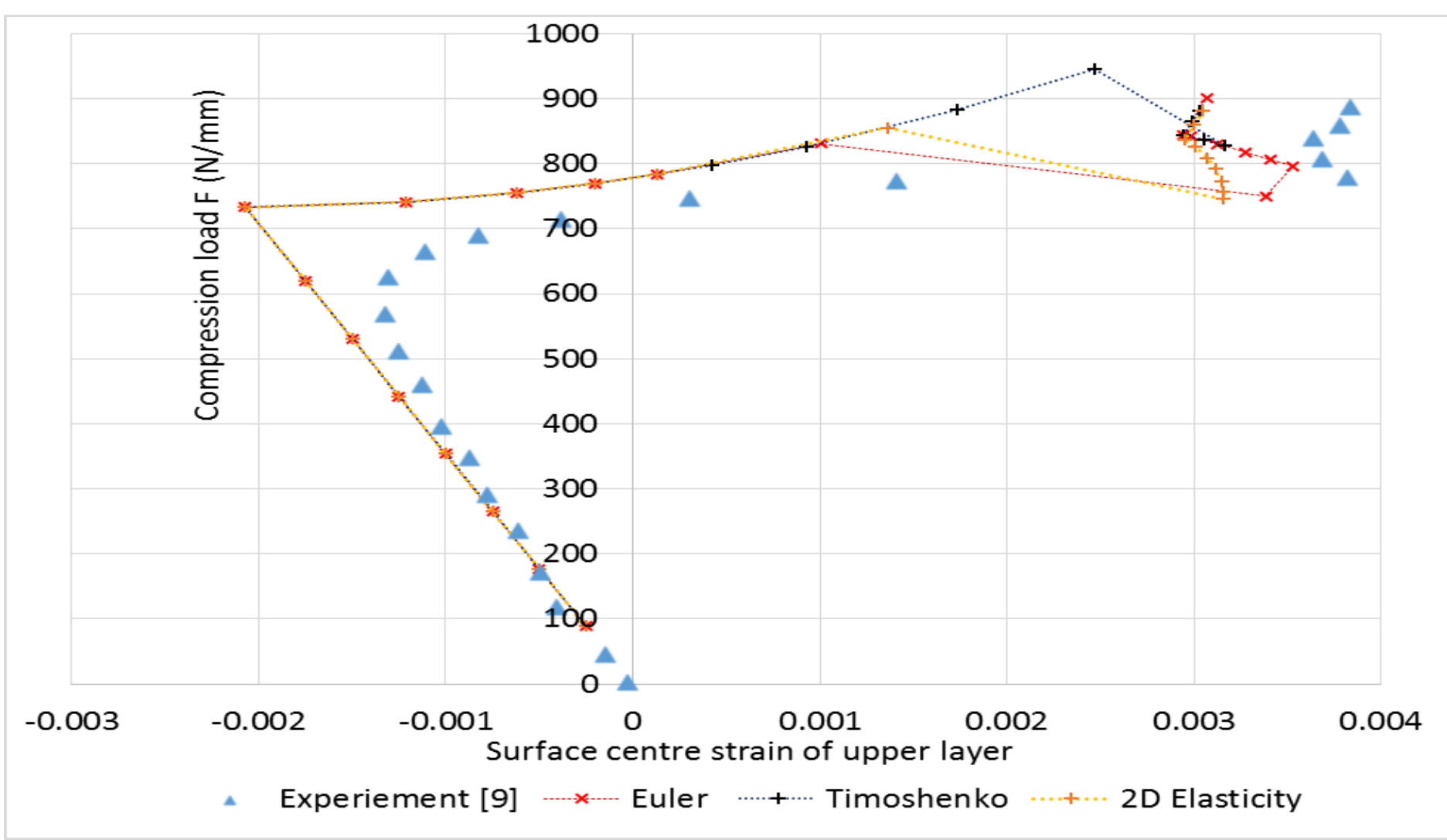




\section{Experimental Validation}

- Fig. 8. Experimental validation of the analytical prediction for case 2 panel

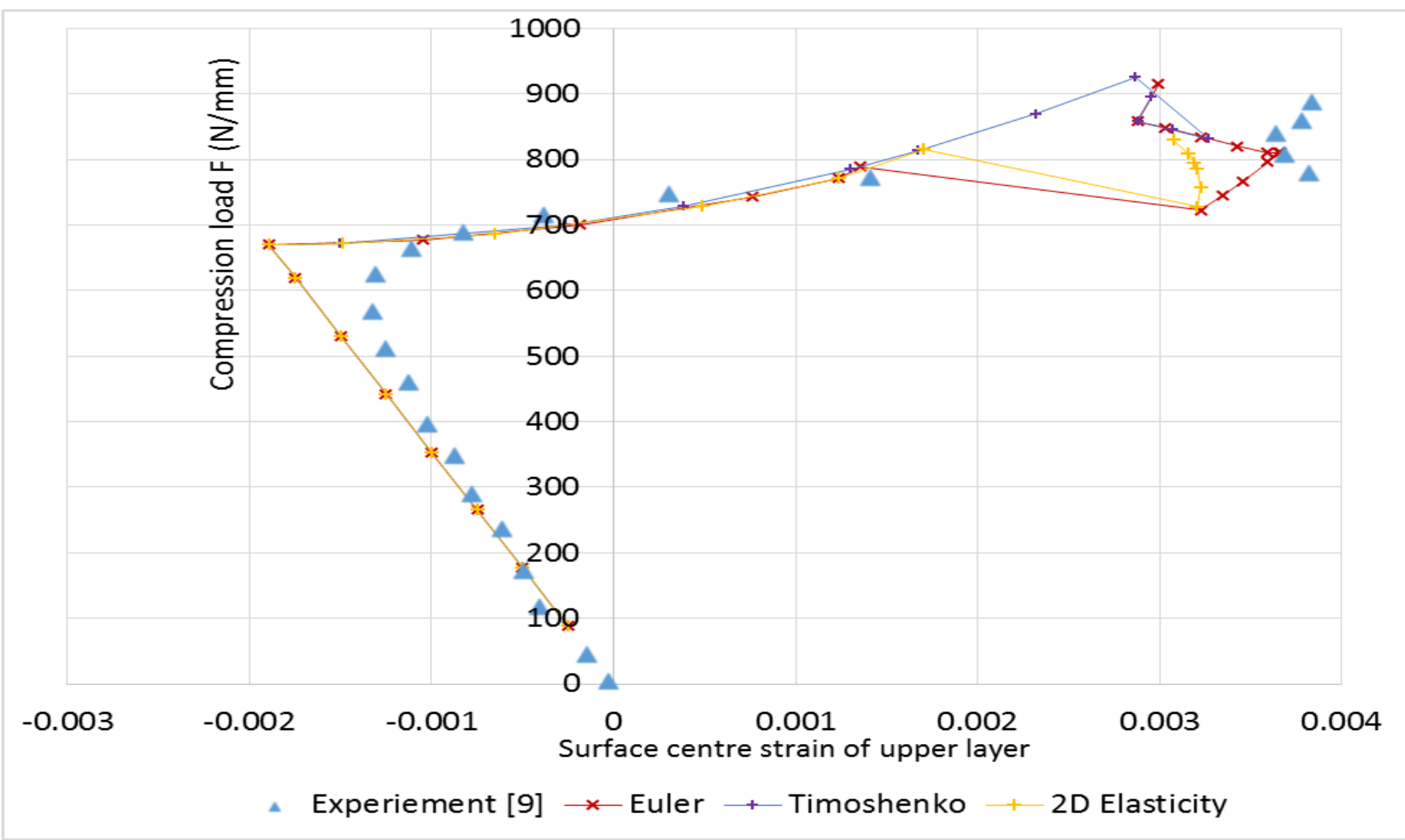




\section{Conclusions}

- Present analytical formula gives accurate calculation of the total ERR.

- Wang-Harvey Euler beam mixed-mode partition theory also governs the propagation of postlocal buckling driven delamination.

- Further validations are necessary. 
Thank you very much and questions are welcome! 